\title{
Carnets
}

Revue électronique d'études françaises de l'APEF

Deuxième série - 12 | 2018

Théorie Mimétique et Études Littéraires

\section{Hagiographie et théorie mimétique}

La Lettre des martyrs de Lyon et Vienne d'Eusèbe de Césarée

\section{Julia Sei}

\section{(2) OpenEdition}

Journals

Édition électronique

URL : http://journals.openedition.org/carnets/2470

DOI : $10.4000 /$ carnets. 2470

ISSN : 1646-7698

Éditeur

APEF

Référence électronique

Julia Sei, « Hagiographie et théorie mimétique », Carnets [En ligne], Deuxième série - 12 | 2018, mis en ligne le 19 janvier 2018, consulté le 19 avril 2019. URL : http://journals.openedition.org/carnets/2470 ; DOI : $10.4000 /$ carnets. 2470

Ce document a été généré automatiquement le 19 avril 2019

\section{(c) (i) \&}

Carnets est mis à disposition selon les termes de la licence Creative Commons - Atribution - Pas d'utilisation commerciale 4.0 International. 


\title{
Hagiographie et théorie mimétique
}

\author{
La Lettre des martyrs de Lyon et Vienne d'Eusèbe de Césarée
}

\section{Julia Sei}

1 La Lettre des chrétiens de Vienne et de Lyon à leurs frères d'Asie et de Phrygie est l'un des premiers récits de persécution contre l'Église chrétienne. Cette lettre, transmise par Eusèbe de Césarée, relate comment, en l'absence du gouverneur, une foule enragée provoqua le massacre de la communauté chrétienne de Lyon en 177. Les textes hagiographiques sont rarement analysés dans une perspective girardienne. Pourtant, les théories de Girard sont éclairantes et révèlent l'ampleur de la violence archaïque et sacrificielle. Les chrétiens de Lyon et de Vienne correspondent parfaitement à la description girardienne du bouc émissaire et réunissent tous les stéréotypes de persécutions que Girard décrit dans son ouvrage éponyme (Girard, 1982). Ces premiers martyrs chrétiens sont des « victimes idéales » au sens girardien. La Lettre des chrétiens de Lyon et Vienne nous fournit de précieux éléments sur le mimétisme de la violence et l'analyse qui suit nous permettra de mieux appréhender les mécanismes de persécution.

2 Les causes exactes de la persécution ne sont pas identifiées par l'auteur. Cependant, certains commentateurs ont suggéré que les auteurs du massacre étaient les citoyens les plus importants de la ville de Lyon (Frend, 1965). En 176-177, l'Empereur et le Sénat décidèrent de soulager les riches propriétaires des provinces de l'Empire de certains frais des jeux gladiatoriaux - les munera - en leur permettant d'utiliser des condamnés à mort à la place des gladiateurs, pour six aurei par tête, soit un dixième du prix normal. Ce décret impérial nous est parvenu grâce à deux documents : une table de bronze, trouvée en 1888 près de Séville et à présent conservée au musée de Madrid, et une plaque de marbre trouvée en 1906 à Sardes (Piganiol, 1975). Sur l'inscription de Séville a été conservé un long discours prononcé par un sénateur gaulois, dans lequel celui-ci fait référence à l'usage, dans sa province, des trinqui, des criminels condamnés à mort. Le sénateur stipule qu' "à cause d'une antique coutume de rituel sacré [les trinqui] sont attendus avec impatience dans les villes des plus belles provinces gauloises ${ }^{1} »$ (Frend, 1965: 5). Là où l'oraison de Sardes suggère une condamnation ferme des jeux où figurent les trinqui, le texte de Séville justifie l'usage gaulois. Cette catégorie de condamnés à mort fait donc office de sacrifice humain (Piganiol, 1975). Or, les mises à morts sanglantes étaient 
particulièrement populaires dans le sud de la Gaule. Le supplicié était une victime offerte aux dieux et pouvait se substituer aux sacrifices faits en l'honneur du dieu gaulois Teutatès (Jullian, 1971). Entre les gladiateurs et les victimes, la différence n'était donc pas très grande et les Romains assuraient aux dieux du pays les offrandes ancestrales ${ }^{2}$. L'historien Camille Jullian précise que l'usage persista en Gaule de couper la tête des ennemis sur le champ de bataille et de les rapporter au son des hymnes de triomphe, suspendues au cou des chevaux. La vie des ennemis était donnée aux dieux en sacrifice et les hommes gardaient pour eux la tête (Jullian, 1971: 140). Cette coutume de trancher la tête des ennemis sacrifiés pourrait justifier, selon André Piganiol, l'usage du terme de trinci ${ }^{3}$ pour les condamnés à morts sacrifiés pendant les jeux. Pour les autorités gauloises, il y avait un triple avantage à tuer les chrétiens : elles se débarrassaient d'une frange potentiellement dangereuse de la population et sacrifiaient aux dieux tout en réduisant le coût des munera.

Dans la lettre, nous apprenons que les chrétiens sont d'abord «écartés des maisons, des bains, de la place publique » puis on leur interdit absolument «de paraître en quelque lieu que ce [soit] » (Eusèbe de Césarée, 1955 : 7). Mais cette isolation est insuffisante; les païens furieux multiplient les sévices contre les chrétiens, ces derniers sont hués, frappés, lapidés, avant d'être emmenés sur le forum et interrogés par le tribun et les premiers magistrats de la ville. Puis, après avoir confessé leur foi, ils sont emprisonnés jusqu'à l'arrivée du légat. Le texte d'Eusèbe montre que la haine populaire est progressive: les chrétiens sont d'abord bannis de certains espaces publics, puis de tous les espaces publics, après quoi ils sont battus et finalement emprisonnés. Une recherche systématique des chrétiens commence alors : "Chaque jour on arrêtait ceux qui en étaient dignes, pour compléter le nombre des martyrs. Ainsi furent emprisonnés tous les croyants zélés des deux Églises, ceux sur qui principalement reposaient les affaires de notre pays » (idem: 9).

Avec le retour du légat, le sort des chrétiens est soumis à un procès public et les autorités utilisent tous les moyens imaginables pour s'assurer des abjurations. Les esclaves païens appartenant aux prisonniers sont arrêtés et menacés de torture, ils sont contraints de déclarer que leurs maîtres avaient commis des actes d'inceste et de cannibalisme :

Ces gens, par une ruse de Satan, furent effrayés par les supplices qu'ils voyaient souffrir aux saints et, poussés en cela par les soldats, ils nous accusèrent faussement de nous livrer à des festins de Thyeste et à des incestes semblables à ceux d'Ædipe, et de faire ce qu'il ne nous est pas permis de dire ni même d'imaginer (Eusèbe de Césarée, 1955 : 9-10).

Dès lors, la rage de la foule est telle qu'il est impossible de l'apaiser :

Ces bruits se répandirent pourtant et tous entrèrent contre nous dans une colère de fauves, si bien que quelques-uns, qui tout d'abord avaient été modérés à cause de nos relations familières avec eux, se montraient alors violemment hostiles et grinçaient des dents contre nous (Idem : 10).

Comme le texte le rappelle, la procédure judiciaire semble biaisée : les autorités usent de la violence et les chrétiens sont arrêtés sans l'autorisation du légat. C'est véritablement la foule qui semble posséder le pouvoir judiciaire. Les chrétiens sont soumis à la torture mais leur force et leur endurance sont saisissantes. Le courage du chrétien Sanctus est "plus qu'humain » (Eusèbe de Césarée, 1955 : 10). À force d'être torturé, son corps cesse d'avoir forme humaine, mais il semble ne plus se soucier des contingences terrestres et sa réponse à chaque question posée par les autorités romaines reste la même: «je suis chrétien $»^{4}$. Cette réponse enrage les païens de plus belle. Le reste du récit relate 
comment ceux qui ont refusé de renier leur foi sont torturés, tués, et leurs dépouilles jetées dans le Rhône.

\section{Analyse}

7 Selon Girard, la crise sociale (épidémie, famine, pauvreté) est très souvent interprétée comme un châtiment divin par la multitude (Girard, 1982). Pour écarter le fléau, il faut découvrir les coupables et les « dévouer » à la divinité5. S'il y a fléau, les préjugés qu'une société entretient à l'égard de certaines minorités peuvent s'exacerber. En effet, les persécuteurs sont toujours convaincus qu'un petit groupe d'individus peut être extrêmement dangereux pour la société entière, même si ce groupe est relativement faible. Dans certains cas, la crise peut être produite par des accusations qui surexcitent la foule et polarisent l'appétit persécuteur sur ceux qui rompent l'ordre de la société. Une fois cet appétit ouvert, les rumeurs les plus absurdes sont acceptées comme des faits (Girard, 1982).

Les chrétiens des $\mathrm{i}^{\mathrm{e}}$, $\mathrm{ii}^{\mathrm{e}}$ et iiie siècles étaient régulièrement victimes de " pogroms ", qui ne cessèrent véritablement qu'au début du iv $v^{e}$ siècle lorsque l'Empereur Constantin imposa le christianisme comme religion d'État (Carrié, 2005). Ces pogroms étaient toujours justifiés : il était légitime de châtier les chrétiens puisqu'ils étaient responsables de « tous les désastres publics ${ }^{6}$. Cette culpabilité supposée des chrétiens se retrouve chez de nombreux auteurs de l'Antiquité 7 . Saint Augustin, dans la Cité de Dieu, rappelle le proverbe: "Il ne pleut pas, c'est de la faute des chrétiens" (Augustin, 2000: 50). L'accusation était si courante qu'elle était même devenue un dicton populaire. Les circonstances qui favorisent ces phénomènes de violence varient, mais ils sont toujours vécus par la communauté persécutrice comme une «perte radicale du social », la fin des règles et des différences qui définissent les ordres culturels.

Girard décrit trois stéréotypes de persécution dans son ouvrage Le Bouc Émissaire. Il désigne les persécutions collectives, qui sont des actes de violence stimulés par la haine de la foule. Le premier stéréotype décrit par Girard concerne la grande uniformité qui caractérise une société en crise et l'indifférenciation du culturel: les différences hiérarchiques et sociales qui régissent la société (respect pour les aînés, piété filiale, etc.) sont effacées par la violence et mènent à la plus grande confusion. Le deuxième stéréotype concerne le type de crimes que la victime est censée avoir commis, crimes qui sont toujours "fondamentaux " car ils s'attaquent aux racines de l'ordre social et le détruisent entièrement. Le troisième stéréotype concerne la sélection des victimes, qui peut être due au hasard, mais qui obéit souvent à un schéma prédéfini. Comme nous allons le démontrer, les martyrs de Lyon et de Vienne correspondent aux trois stéréotypes de persécution proposés par Girard et peuvent être considérés comme des « boucs émissaires » en puissance, des victimes idéales.

\section{Stéréotype $n^{\circ} 1$. La crise sociétale : uniformité et monotonie}

Une société en crise est caractérisée par sa grande uniformité. Les institutions sont affaiblies et la foule est susceptible de prendre l'ascendant sur les autorités fragilisées. La violence et l'aveuglement mènent à la confusion à l'intérieur de la communauté et 
produit une grande uniformité. Cette notion peut paraître paradoxale à première vue. En temps de crise, les gens s'opposent les uns aux autres, ce qui devrait renforcer les différences culturelles. Cependant, Girard remarque qu'une société en crise est en fait grandement uniforme et conduit à la prédominance du «même ${ }^{8}$. Il y a bien réciprocité, mais dans les échanges négatifs, comme les insultes et la colère.

11 Les périodes de crise favorisent la formation des masses, puisque les institutions normales sont affaiblies ${ }^{9}$. Le rassemblement spontané de ces masses peut exercer une influence décisive sur des institutions fragilisées et même, dans les cas les plus extrêmes, les remplacer complètement. C'est ce qui se passe à Lyon et Vienne, puisque les chrétiens sont arrêtés sans la permission du légat. C'est bien la foule qui semble posséder le pouvoir judiciaire. Les autorités semblent simplement suivre la volonté de la foule enragée. Il existe un appel obscur de la foule, qui la mobilise, qui la transforme en mob: la mobilisation se fait alors contre l'ennemi désigné. Des communautés que tout oppose d'ordinaire sont alors susceptibles de s'allier contre un ennemi commun, comme c'est le cas dans le martyre de Polycarpe, puisque juifs et païens s'unissent contre Polycarpe et demande aux autorités de lâcher un lion sur le vieil évêque (Ignace d'Antioche, 2007 : 225). Les chrétiens étaient régulièrement accusés d'athéisme (deos non colere) par les païens car ils ne reconnaissaient d'autres dieux que le leur (De Ste Croix, 1963). De manière significative, on retrouve ces accusations d'athéisme chez les chrétiens envers les païens, notamment lorsque l'évêque Polycarpe accuse la foule d'athéisme. (Ignace d'Antioche, $2007: 223$ ).

Girard soutient que le pouvoir de la violence peut facilement écarter la raison et se propage aussi rapidement que la peste. Cet aveuglement causé par la haine peut devenir si intense que, parfois, le véritable «ennemi » ou "adversaire » est perdu de vue. L'effondrement des institutions élimine les différences hiérarchiques et donne à la société un aspect monotone et monstrueux: une fois que les accusations d'inceste et de cannibalisme sont rendues publiques - renvoyant les chrétiens à leur inhumanité - c'est bien la foule qui semble se transformer en bête furieuse : les païens entrent dans une colère de fauves et grincent des dents ${ }^{10}$. La perte d'humanité de la multitude païenne est omniprésente dans le texte d'Eusèbe ${ }^{11}$. Il y a ainsi une perte de l'ordre social mise en lumière par la disparition des règles et des différences qui d'ordinaire distinguent une société saine.

\section{Stéréotype $n^{\circ} 2$ : Inceste et cannibalisme}

Les crimes supposés de la victime sont toujours «fondamentaux »: ils s'attaquent à la base de l'ordre culturel, comme la famille ou les différences hiérarchiques (Girard, 1982). Les chrétiens étaient accusés d'attaquer les règles sociales, religieuses et sexuelles les plus élémentaires. En effet, les premiers chrétiens attaquaient les fondations de la société romaine en refusant l'existence des dieux païens, en refusant de consommer la viande sacrificielle ${ }^{12}$ et en récusant la divinité de l'Empereur, comme nous venons de voir. Or, à la fin des années 170, Marc-Aurèle fit un retour victorieux de sa campagne militaire sur le Danube et en Orient, retour qui donna lieu à de grandes réjouissances auxquelles les chrétiens refusèrent certainement de s'associer. En 176, des cérémonies tauroboliques en l'honneur de l'Empereur sont attestées, notamment en Aquitaine et en Narbonnaise (Le Glay, 1978). L'intransigeance des chrétiens et leur refus catégorique de participer aux sacrifices les condamnent aux foudres de l'auctoritas romaine (De Ste Croix, 1963 ; Carrié, 
2005 ; Frend, 1965). Dès que les chrétiens étaient assez nombreux dans une ville, ils formaient une assemblée ou ecclesia, et désignaient un évêque. Ils se ressemblaient fréquemment dans des espaces clos et privés - chez un autre chrétien, par exemple. La nature essentiellement privée de leur culte, qui tranchait avec le faste public des rites romains, éveillait et nourrissait les suspicions (Frend, 1965). Comme le souligne JeanMichel Carrié, la plupart des chrétiens et des païens étaient convaincus de la réalité et de l' efficacité des sacrifices: il était donc difficile de faire des gestes symboliques ou de donner aux sacrifices une interprétation allégorique, même pour sauver sa vie (Carrié, $2005: 142$ ).

Précisons que dans l'Empire romain, une grande diversité religieuse existait et, même s'il serait anachronique de parler de "tolérance " religieuse, une multitude de divinités coexistaient dans la cité (Turcan, 1978). Durant le $\mathrm{II}^{\mathrm{e}}$ siècle après J.-C., les cultes orientaux, notamment celui d'Isis ou de Mithra, étaient très en vogue dans les milieux aristocratiques (Cumont, 2016). En période de guerre, notamment, Rome ne refusait pas l'aide que pouvait lui apporter des dieux étrangers. Dans l'empire Romain, le sacrifice était au cœur de la piété publique, à la fois civique et religieuse. Les persécutions dont furent victimes les chrétiens étaient donc dues à l'opiniâtreté de ces derniers et à leur refus systématique du compromis, qui troublait l'ordre de l'Empire (Carrié, 2005). Ce comportement extrême de certains chrétiens les rendit suspects aux yeux des citoyens romains.

Dans La violence et le Sacré, Girard note que la violence collective, comme le lynchage ou les pogroms, est toujours justifiée par des accusations d'inceste, de parricide ou d'infanticide. Ces crimes, par leur nature, sont une trahison des règles les plus élémentaires de la société humaine (De Ste Croix, 1963). Les individus coupables de telles aberrations méritent l'expulsion ou la mort. Or, les chrétiens de Lyon et de Vienne sont accusés de cannibalisme et d'inceste. Ce types d'accusations contre les chrétiens étaient fréquents dans l'Antiquité tardive comme en témoigne l'Octavius de Minucius Felix, un apologiste chrétien du deuxième siècle. Ce texte, qui se présente sous forme de dialogue entre des amis chrétiens et païens, est une défense du christianisme. Au début du texte, Caecilius, un païen cultivé, fait l'apologie de la religion romaine et décrie les mœurs des chrétiens ainsi :

À jour fixe ils se réunissent pour banqueter avec tous leurs enfants, sœurs et mères, gens de tous sexes et de tous âges. (...) Ils enveloppent dans l'impudeur des ténèbres les étreintes de leur passion répugnante, au hasard du sort, tous également incestueux, sinon en acte, du moins par complicité, puisque leurs vœux unanimes convoitent tout ce qui peut se produire dans les actes individuels (Minucius Felix, $1974: 13-14)$.

Cette accusation d'inceste est précédée de manière significative d'une accusation de cannibalisme (Minucius Felix, 1974: 13). Le cannibalisme dont les chrétiens étaient accusés participe à cette perte des différences. Cependant, ici, il ne s'agit plus de différences culturelles mais humaines. C'est bien le cas dans les martyrs de Lyon, puisque les accusations de cannibalisme distinguent les chrétiens des païens : ces derniers ne considèrent plus les chrétiens comme véritablement humains et ils sont alors susceptibles d'être sacrifiés. Le nom donné au pain consacré de l'eucharistie - l'hostie - est dérivé du terme latin hostia « la victime offerte aux dieux » (Chavaud, et al., 2012: 8; Tannahill, 1975 : 58). Or, dans le nouveau monde chrétien, le Christ en tant que victime humaine et l'hostie représentant son corps étaient interprétés de manière littérale (Tannahill, 1975 : 58-59). 
17 L'inceste détruit la structure familiale, c'est-à-dire la distinction entre membres de la même famille. Avec l'inceste, l'abolition violente des différences est achevée. Jean-Pierre Vernant décrit cette perte violente des différences dans son ouvrage Ambiguïté et renversement. Sur la structure énigmatique d'Ædipe roi : « Le parricide et l'inceste constituent (...) une atteinte aux règles fondamentales d'un jeu de dame où chaque pièce se situe, par rapport aux autres, à une place définie sur l'échiquier de la Cité » (Vernant, 1972 : 127). Ces crimes sont donc une atteinte fondamentale à l'ordre de la cité. En considérant ces éléments, on comprend mieux ces accusations: lorsqu'elles deviennent publiques, la violence s'intensifie et même les citoyens « modérés » perdent la raison.

\section{Stéréotype $n^{\circ} 3$ : la sélection victimaire}

18 La foule peut choisir une victime complétement au hasard, mais ce n'est pas forcément le cas. Selon Girard, la plupart des sociétés soumettent leurs minorités à certaines formes de discriminations, et parfois même à des persécutions. Ainsi, il y a des signes universels pour la sélection des victimes. L'autre, l'étranger, est souvent accusé de crimes fondamentaux. Or, la communauté chrétienne de Lyon avait un large contingent étranger : la ville de Lugdunum et la vallée du Rhône étaient d'importantes plateformes commerciales, habituées à traiter avec les peuples méditerranéens depuis l'ère mycénienne (Frend, 1965). Les influences asiatiques et orientales étaient donc importantes dans cette région de Gaule (Turcan, 1978). De plus, les noms des martyrs préservés dans le texte d'Eusèbe sont tous asiates: Pothin, le vieil évêque, était probablement un immigrant et Irénée avait été élevé à Smyrne. Attale, un chrétien important de la ville, était un citoyen romain, de Pergame. Alexandre, un autre personnage d'influence, était un physicien phrygien (Quentin, 1983).

19 En plus des différences culturelles et religieuses, les différences physiques (la maladie, la folie, les malformations génétiques, les handicaps en général) ont tendance à polariser les persécuteurs. L'anormalité peut fonctionner comme un critère de sélection pour les persécutions. Le mot même d'anormal, comme le mot peste au Moyen-Âge, a quelque chose de tabou, de sacré au sens originel du terme. Les caractéristiques extrêmes provoquent la destruction collective à certains moments. De fait, Eusèbe dans son Histoire Ecclésiastique, décrit constamment la stupeur des païens face à la détermination des chrétiens dans leurs martyres. L'arène s'émerveille de l'endurance des martyrs. C'est peut-être pour rendre cet aspect plus explicite encore que les Actes des Martyrs semblent se focaliser sur les membres les plus vulnérables de la société. Blandine, dans les Martyrs de Lyon, est une esclave, et elle est accompagnée d'un adolescent de quinze ans, Pontique. Elle est décrite comme apparaissant aux yeux des hommes: "sans beauté, simple, méprisable » et les chrétiens craignent « qu'elle ne soit pas capable, à cause de sa faiblesse physique, de faire avec assurance sa confession de foi » (Eusèbe de Césarée, 1955: 10). Cependant, elle endure des périodes de torture prolongées et son endurance est telle que ce sont ses bourreaux qui doivent s'avouer vaincus. Perpétue, dans la Passion des saintes Perpétue et Félicitée, est une jeune mère qui allaite encore ; Félicitée, son esclave, est une très jeune fille. Les textes soulignent même les infirmités physiques de ceux qui ont un statut important dans la communauté chrétienne. Pothin, l'évêque de Lyon, a «plus de quatre-vingt-dix ans ", il est « très faible de corps et [peut] à peine respirer à cause de [sa] faiblesse physique » (Eusèbe de Césarée, 1955 : 13-14). Ces mêmes qualités qui suscitent l'admiration des païens provoquent une soif de revanche allant au-delà de la mort des 
chrétiens. Ce sont tous, d'une certaine manière, des victimes idéales: d'origines étrangères, faibles, parfois esclaves, ils portent tous la marque victimaire.

Comme nous pouvons l'observer avec cet exemple, l'effondrement de l'ordre sacré et des tabous a pour effet de libérer les tendances agressives jusqu'alors enfouies, dormantes, qui commencent à se propager en toutes directions. Cette éruption de la violence renforce la crise des concepts culturels et religieux. C'est précisément ce qui se passe avec les chrétiens de Lyon : ils transgressent l'ordre sacré de Rome en refusant de sacrifier aux dieux et à l'Empereur et les accusations d'inceste et de cannibalisme libèrent la violence mimétique qui mène à la crise sacrificielle.

\section{Les martyrs chrétiens, boucs émissaires et pharmakoi ?}

Revenons brièvement sur la signification originelle du bouc émissaire. Ce rappel nous permettra de mieux comprendre l'aspect proprement sacrificiel du massacre de 177, au sens girardien.

Le rite originel du bouc émissaire, tel qu'il est décrit dans le Lévitique 16, versets 1-34 est un rite d'expiation, censé détruire les impuretés du peuple d'Israël (Watts, 2007). Pendant la cérémonie, deux boucs sont présents : l'un, le bouc émissaire, est épargné et envoyé dans le désert après avoir symboliquement pris sur lui les péchés du peuple d'Israël ; l'autre est sacrifié à Yahvé. Les transgressions et les péchés des Israélites sont donc transférés collectivement sur le bouc émissaire. Pendant ce rituel, l'autre bouc est offert à Dieu et le sang sacrificiel de cette deuxième offrande doit purifier le sanctuaire. Le pardon de Dieu, qui n'est offert qu'à la fin du processus expiatoire, ne peut être donné que si le rituel est accompli de façon satisfaisante. Le passage suivant décrit une scène de Yom Kippour, où le grand prêtre des Hébreux préside la cérémonie du bouc émissaire :

Il recevra de l'assemblée des enfants d'Israël deux boucs destinés à un sacrifice pour le péché et un bélier pour un holocauste. Après avoir offert le taureau du sacrifice pour son propre péché et fait le rite d'expiation pour lui et pour sa maison, Aaron prendra ses deux boucs et les placera devant Yahvé à l'entrée de la tente de réunion. Il tirera les sorts pour les deux boucs, attribuant un sort à Yahvé et l'autre à Azazel. Aaron offrira le bouc sur lequel est tombé le sort « À Yahvé » et en fera un sacrifice pour le péché. Quant au bouc sur lequel est tombé le sort « À Azazel » on le placera vivant devant Yahvé pour faire sur lui le rite d'expiation, pour l'envoyer à Azazel dans le désert (Le Lévitique, 1958 : 16).

L'élimination du bouc émissaire exprime l'élimination du danger et la précision du rite indique qu'une réelle menace était perçue. Dans la tradition juive, Azazel est un démon vivant dans le corps d'un bouc (Dawson, 2013; Pinker, 2007). Dans ce rituel, même si le bouc n'est pas tué, il est expulsé de la communauté. Le but de cette cérémonie est donc de prouver au peuple d'Israël que ses péchés ont été lavés. La confession d'une faute commune sur la tête du bouc correspond à une agression rituelle pendant laquelle la communauté entière se lave de ses péchés. Ce bouc dont la vie est épargnée est devenu la figure par excellence de la victimisation et n'est plus à présent un rituel religieux mais une métaphore séculaire (Dawson, 2013).

Mary Douglas, dans son ouvrage De la souillure, souligne que les rites de pureté et d'impureté produisent l'unité du groupe. Les règles de pureté rituelles gardent les choses et les membres de la société à leur place. De plus, les concepts d'impureté, de souillure, 
sont des marqueurs d'identité sociale et servent à définir l'identité des individus (Douglas, 1996). Ainsi, les membres d'une société se définissent par différents degrés de pureté. Cette contagion de l'impur sert à créer une hiérarchie au sein de la communauté. Selon Douglas, les rites purificateurs ont pour but de créer l'unité au sein de la communauté et ce sont ces règles qui permettent à la société d'exister «être saint, c'est être entier, être un ; la sainteté, c'est l'unité, l'intégrité, la perfection de l'individu et de ses semblables.» (Douglas, 1981: 73). Par conséquent, les individus jugés «moins purs » sont écartés en toute légitimité.

Les rites d'expiation sont donc intimement liés à l'ordre et à la pureté. Le rite hébraïque du bouc émissaire offre des points de convergence avec celui, hellénistique, du pharmakos. Dans l'antique Athènes, des cas de sacrifices humains ont été relevés (Eck, 2011; Jeanmaire, 1991; Séchan, 1966). Ces pratiques étaient perpétrées sous la forme du pharmakos: celui-ci vivait aux frais de la cité et était occasionnellement sacrifié, notamment en périodes de crises (Eck, 2011). Au V $\mathrm{V}^{\mathrm{e}}$ siècle avant J.-C., le sacrifice humain particulièrement cruel du pharmakos ne survivait que dans une version édulcorée, mais il n'en avait pas toujours été ainsi et «aux confins de l'Empire, à Marseille ou à Abdère, nous entendons parler de pharmakoi jetés à la mer ou lapidés " (Jeanmaire, 1991 : 229). À Athènes, un processus d'expulsion moins sauvage survivait: le $6 \mathrm{du}$ mois Thargélion (mai), on purifiait la ville en escortant à travers les rues deux pharmakoi « on les frappaient à coups de branches de figuiers et de tiges d'oignons marins, puis on les expulsait de la ville, pour écarter toutes les souillures dont on les avait ainsi chargés » (Séchan, 1966: 205). Le pharmakos - qui signifie filtre ou médicament - a un statut ambivalent puisqu'il est à la fois remède et poison (Derrida, 1972). D'autres versions du rite du pharmakos mentionnent la promenade rituelle à travers les rues de la cité pour que l'individu absorbe les souillures de la ville (Eck, 2911). Ensuite, on lui frappe les parties génitales, puis on le brûle et on répand sa cendre au gré du vent. Ce pharmakos est, du fait de son statut social (car il est bien souvent esclave) en dehors de la société (Girard, 1972).

Dans ces deux rites primitifs, celui du bouc émissaire et celui du pharmakos, l'objet pollué est expulsé ou détruit par l'ensemble de la communauté. La violence est canalisée par l'intermédiaire du rite et permet de contenir la violence. Le rite évite donc que la violence ne se disperse de façon désordonnée au sein de la communauté. Le sacrifice est une violence sans risque de vengeance. Les victimes chrétiennes à Lyon et Vienne pouvaient assumer ce rôle de pharmakos : menaçant et dangereux vivant mais qui restaure l'ordre social une fois mort. Nous suggérons qu'une sorte de rite sacrificiel opère dans l'arène, où les martyrs chrétiens deviennent victimes émissaires. Les festivités pendant lesquelles les chrétiens sont mis à mort, les munera, retiennent un caractère rituel car elles commémorent la crise sacrificielle et représentent à la fois l'acmé et la conclusion du festival. Les ornements imaginaires des martyrs - leurs blessures - contribuent à faire de leur mort un spectacle ${ }^{13}$. La crise et la violence sacrificielles sont célébrées de façon théâtrale car elles sont un prélude à la restauration de l'ordre.

Le fait que les chrétiens soient mis à mort dans l'amphithéâtre et que certains chrétiens soient « réservés » pour une fête particulière n'est pas anodin et participe de cette crise sacrificielle ${ }^{14}$. L'unité de la communauté païenne est à ce moment très étroite, tous sont liés contre l'ennemi qui menace l'ordre sacré. En tuant les chrétiens de manière spectaculaire, l'ordre culturel est vivifié car l'expérience fondatrice est répétée sous les yeux des païens. 
L'expulsion des chrétiens de tous les espaces publics à Lyon, avant leur martyre, peut être compris comme un rite d'expulsion sacrificiel correspondant à une période d'anarchie et de violence incontrôlée. Cette expulsion sert à purifier la cité. Des termes tels que " crier ", « furieux ", « en rage ", "grincer des dents », sont récurrents pour décrire la foule : symboliquement, c'est la communauté païenne dans son ensemble qui participe au rituel de purification. L'amphithéâtre romain devient le tabernacle sur lequel les victimes sont sacrifiées et devient le lieu où la violence se canalise. Il est notable que pour Girard, crier, frapper des objets les uns contre les autres ou agiter des objets dans l'air sert à chasser les mauvais esprits d'un lieu. Ainsi, les païens ne montreraient pas seulement leur profonde haine des chrétiens, ils manifesteraient également une peur de l'inconnu et des mauvais esprits. C'est un exemple du fort aspect mimétique de la violence. Violence et imitation sont donc deux faces d'une même médaille: la violence est contagieuse car chacun désire imiter la réaction de la foule. La violence et l'imitation se complètent, et la seule façon de canaliser cette contagion de la violence est d'expulser ou de tuer la victime sacrificielle.

\section{Remarques conclusives}

Cette analyse détaillée des martyrs de Lyon nous donne les clés nécessaires pour comprendre le processus victimaire tel qu'il est décrit par René Girard. En canalisant sa haine contre les chrétiens, la communauté païenne de Lyon élimine ses instincts violents et pense ainsi se débarrasser des éléments impurs qui pervertissent la société. Ainsi, la mort des chrétiens ramène la paix et l'ordre et permet d'éliminer les souillures de la communauté païenne. D'une part, la mise à mort apparaît comme un acte pie, d'autre part, c'est une véritable fonction religieuse que les exécutés remplissent (Gernet, 1924). Cependant, cette notion de crise et de sacrifice peut être comprise de deux perspectives bien distinctes : la païenne et la chrétienne. La mort humiliante des chrétiens dans l'arène est interprétée, dans la lettre, comme le destin le plus honorable qui soit, et correspond à un retour de l'ordre divin : la mort des chrétiens constitue une " défaite » pour les païens, puisque les martyrs n'ont pas renié leur foi. La lettre utilise abondamment le symbolisme de la virtus romaine pour exprimer la victoire chrétienne. Les martyrs sont considérés comme des "nobles athlètes du Christ ", un pendant ironique aux gladiateurs romains ${ }^{15}$.

La théorie du bouc émissaire de Girard met en lumière le mécanisme de violence archaïque présent dans les martyrs de Lyon. En tuant les chrétiens, les païens restaurent l'ordre universel romain. Les martyrs de Lyon correspondent à la définition girardienne du bouc émissaire et correspondent à ce que Girard a appelé des «stéréotypes de persécution ». Le martyr, en tant que bouc émissaire, canalise la violence collective et permet la fin de la crise sacrificielle. En plaçant la violence au cœur de sa pensée, Girard montre qu'elle joue un rôle important dans toutes les sociétés. Le bouc émissaire devient le processus structurant qui régule les tensions internes d'une société en créant "les différences fondamentales entre profane et sacré, humain et non-humain ». Le bouc émissaire aide à maintenir les distinctions entre les différentes sphères de la société. En analysant ce témoignage de persécution chrétienne dans une perspective girardienne, on découvre de nouvelles voies interprétatives. 


\section{BIBLIOGRAPHIE}

ARNOBE (2010). Contre les gentils. Éd. Bernard Fragu. Paris : Les Belles Lettres.

Augustin (2000). La Cité de Dieu. Éd. Lucien Jerphagnon. Paris : Gallimard.

CARRIÉ, Jean Michel \& ROUSSELLE, Aline (2005). L'Empire romain en mutation : des Sévères à Constantin, 192-337. Paris : Seuil.

CHAVAUD, Frédéric \& GARDES, Jean-Claude \& MONCELET, Christian \& VERnoIs, Solange

(2012). « Introduction générale », in CHAVAUD, Frédéric \& GARDES, Jean-Claude \& MONCELET, Christian \& VERNOIS, Solange. Boucs émissaires, têtes de turcs et souffre-douleur. Rennes : Presses Universitaires de Rennes, pp. 1-13.

COOLEY, Alison (2013). The Cambridge Manual of Latin Epigraphy. Cambridge: Cambridge University Press.

CUMONT, Franz (2015). Les Mystères de Mithra : aux sources du paganisme romain. Rosières en Haye : Camion Blanc.

CYPRIEN (2003). À Démétrien. Éd. Jean-Claude Fredouille. Paris: Cerf.

DAWSON, David (2013). Flesh Becomes Word: a Lexicography of the Scapegoat or, the History of an Idea. East Lansing : Michigan State University Press.

DERRIDA, Jacques (1972). La Dissémination. Paris : Seuil.

DE SAINTE CRoIX, Geoffrey Ernest Maurice, (1963). « Why Were the Early Christians Persecuted? », Past \& Present , no. 26, pp. 6-38.

DOUGLAS, Mary (1981). De la souillure. Paris: Maspero.

DOUGLAS, Mary (1996). « Sacred Contagion », in John F. A. Sawyer. Reading Leviticus: Responses to Mary Douglas. Sheffield: Sheffield Academic Press, pp. 86-106.

ECK, Bernard (2011). «Le pharmakos et le meurtrier », in Véronique Liard. Histoire de crimes et societé. Dijon : Éditions Universitaires de Dijon, pp. 15-29.

EUSÈBE DE CÉSARÉE (1955). Histoire Ecclésiastique. Éd. Gustave Bardy. Paris: Cerf.

FREND, William H. Clifford (1965). Martyrdom and Persecution in the Early Church. Oxford : Blackwell. GERNET, Louis (1924). «Sur l'exécution capitale », Revue des Études Grecques, tome 37, fasc. 172, pp. 261-293.

GIRARD, René (1982). Le Bouc émissaire. Paris : Grasset.

GIRARD, René (1972). La Violence et le Sacré. Paris : Grasset.

JEAnMaire, Henri (1991). Dionysos : histoire du culte de Bacchus. Paris: Payot.

LAST, Hugh (1949). « Rome and the Druids », Journal of Roman Studies, vol 39, pp. 1-5.

LE GLAY, Marcel (1978). « Le culte impérial à Lyon au II ${ }^{\mathrm{e}}$ siècle ap. J.-C. », in Jean Rougé, Robert Turcan. Les Martyrs de Lyon (177). Paris : Éditions du C.N.R.S, pp. 19-31.

LÉVÊQUE, Pierre \& SÉCHAN, Louis (1990). Les Grandes Divinités de la Grèce. Paris : A. Colin. 
Le Lévitique (1951). Éd. Henri Cazelles. Paris : Cerf.

MINUCIUS FELIX (2002). Octavius. Éd. Jean Beaujeu. Paris : Belles Lettres.

Passion de Perpétue et de Félicité (1996). Éd. Jacqueline Amat. Paris : Cerf.

PIGANIOL, André (1975). Recherches sur les jeux romains : notes d'archéologie et d'histoire religieuse. Strasbourg: Librairie Istra.

PINKER, Aaron (2007). « A Goat to Go to Azazel », Journal of Hebrew Scriptures, vol. 7, pp. 1-25.

TANNAHILL, Reay (1996). Flesh and blood: a history of the cannibal complex. London : Abacus.

TERTULLIEN (1998). Apologétique. Éd. Jean-Pierre Waltzing. Paris : Belles Lettres.

TURCAN, Robert (1978). « Les religions orientales à Lugdunum en 177 », in Jean Rougé, Robert

Turcan. Les Martyrs de Lyon (177). Paris : Éditions du C.N.R.S, pp. 195-208.

QUENTIN, Henri (1921). « La liste des martyrs de Lyon de l'an 177 », Analecta Bollandiana, n 39, pp. 113-138.

VERNANT, Jean Pierre (1972). « Ambiguïté et renversement, sur la structure énigmatique d'OedipeRoi », in Jean-Pierre Vernant, Pierre Vidal-Naquet. Mythe et Tragédies en Grèce ancienne. Paris :

Maspero, pp. 99-131.

WATTS, James Winston (2012). Ritual and Rhetoric in Leviticus: from Sacrifice to Scripture. Cambridge :

Cambridge University Press.

\section{NOTES}

1. Ma traduction.

2. Les sacrifices humains étaient courants en Gaule jusqu'au Ier siècle avant J.-C. C'est pour cette raison que les autorités romaines avaient banni les rites druidiques. Voir (Last, 1949).

3. Le terme trinci dériverait d'un terme de latin populaire trincare, trancher (Piganiol, 1975 : 71).

4. À chaque nouvelle question, Sanctus répond en latin aux autorités : christianus sum.

5. Girard précise que pendant l'épidémie de peste noire qui ravagea la France en 1349-50, de nombreux juifs furent désignés comme coupables et par conséquent mis à mort. Dans certaines villes, les juifs étaient massacrés avant même l'arrivée de la peste, au seul bruit de sa présence dans les environs (Girard, $1982: 10$ ).

6. Tertullien, dans l'Apologétique, déplore le traitement réservé aux chrétiens (1998:185).

7. Par exemple, Cyprien, À Démétrien, $2003: 73$ ou Arnobe, Adversus Nationem , $2010: 135$.

8. Girard cite l'exemple du moine portugais Francisco de Santa Maria en 1697 : « Dès que s'allume dans un royaume ou une république ce feu violent et impétueux, on voit les magistrats abasourdis, les populations épouvantées, le gouvernement politique désarticulé (...). Tout est réduit à une extrême confusion. » (Girard, $1982: 24)$. Cette escalade de la violence mimétique fait des hommes des doubles.

9. Or, Robert Turcan, dans son ouvrage sur les martyrs de Lyon, souligne que l'Empire romain durant le siècle de Marc Aurèle commençait à souffrir d'une crise financière, économique et politique qui ne pouvait manquer d'avoir une incidence sur la prospérité de la ville de Lyon. (Turcan, 1978 : 207).

10. La multitude païenne se transforme en $\theta$ nó́ov, en bête sauvage.

11. De nombreux passages de la Lettre montrent l'association de la foule païenne à des bêtes sauvages $(10,16)$ : «l'inhumanité des païens », « peuple en délire ». La foule est furieuse, en rage, et réclame la mort des chrétiens en hurlant. Cette bestialité augmente encore une fois les 
chrétiens morts : «Surexcitées par la bête féroce, ces tribus sauvages et barbares étaient en effet difficiles à apaiser et leur démesure prit un autre tour particulier contre les cadavres. Car leur défaite ne leur faisait pas baisser les yeux - ils n'avaient plus de raison humaine - mais elle enflammait davantage leur colère comme celle d'un fauve » (21).

12. Ce refus de consommer la chair des animaux sacrifiés est visible dans la lettre (12-13).

13. "Les uns en effet s'avançaient souriants ; beaucoup de gloire et de grâce se mêlaient sur leur visage, de sorte que même leurs liens les enveloppaient d'une parure seyante, comme pour une mariée dans ses ornements frangés et brodés d'or ; en même temps, ils répandaient la bonne odeur du Christ et quelques-uns croyaient qu'ils s'étaient oints d'un parfum mondain » (1955: 15).

14. "César répondit qu'il fallait mettre les uns à la torture mais libérer ceux qui renieraient. La fête solennelle du pays - elle est très fréquentée et on $\mathrm{y}$ vient de toutes les nations - ayant commencé de se tenir, le gouverneur fit avancer les bienheureux au tribunal d'une manière théâtrale, pour les donner en spectacle aux foules.» (1955: 18). Cette fête réunissait à Lyon, le 1er août, les délégués des trois Gaules et de Narbonnaise. Elle se prolongeait pendant plusieurs semaines.

15. La lettre utilise abondamment le vocabulaire martial habituellement employé pour décrire un combat de gladiateurs. Nous avons relevé de nombreuses occurrences dans le texte : Maturus est un "généreux athlète» (1955: 10), la maîtresse de Blandine, une «combattante parmi les martyrs» (1955: 10). En décrivant le supplice des chrétiens dans l'arène, la lettre stipule « Il fallait bien que ces athlètes généreux soutinssent des combats variés et, après avoir remporté la grande victoire, reçussent la grande couronne de l'incorruptibilité ». (1955: 15). Blandine, torturée maintes fois, remporte la couronne car elle a revêtue « le grand et invincible athlète, le Christ » $(1955: 17)$.

\section{RÉSUMÉS}

La Lettre des martyrs de Lyon et Vienne, préservée dans l'Histoire Ecclésiastique d'Eusèbe de Césarée, Livre V, décrit une des persécutions les plus cruelles contre la nouvelle Église chrétienne. La lettre relate comment, en l'absence d'un gouverneur, en 177 , une multitude furieuse réclama le massacre de la communauté chrétienne. J'analyserai comment la théorie mimétique révèle de nouveaux aspects sur l'hagiographie en tant que genre littéraire. Les martyrs de Lyon correspondent à la définition girardienne du bouc émissaire et réunissent tous les «stéréotypes de persécutions » établis par Girard. Le martyr canalise la violence collective et régule ainsi les tensions internes de la communauté. Je tenterai d'établir une herméneutique de l'hagiographie dans laquelle la sphère évangélique révèle l'étendue de la violence sacrificielle. Grâce à une lecture girardienne, nous découvrirons un nouvel aspect des textes hagiographiques.

The Martyrs of Lyons and Vienne, preserved in Eusebius' Ecclesiastical History, book V, describes one of the cruelest persecutions to be executed against the early Church. The letter relates how, in the absence of a governor, in 177, an infuriated mob clamored for the massacre of the Christian community. I am aiming to show how mimetic theory casts a new light on hagiography as a literary genre. The Martyrs of Lyons perfectly corresponds to the Girardian definition of the scapegoat, and unites all of Girard's stereotypes of persecution. The scapegoat/martyr is channeling collective violence and thus regulates the community's internal tensions. I will aim 
for a hermeneutics of hagiography in which the evangelic sphere reveals the depth of sacrificial violence. A new aspect of hagiographical texts can be unravelled with a Girardian reading.

31 janvier 2018

INDEX

Keywords : hagiography, persecution, Girard (René), scapegoat, martyr

Mots-clés : hagiographie, persécution, Girard (René), bouc Émissaire, martyr

\section{AUTEUR}

JULIA SEI

Université Paris-Est Créteil

julia.sei[at]hotmail.fr 